\title{
Therapie bei Progression und Rezidiv des Ovarialkarzinoms
}

\author{
W. Meier $\quad$ M. Gropp $\quad$ A. Burges ${ }^{\mathrm{b}} \quad$ H. Hepp

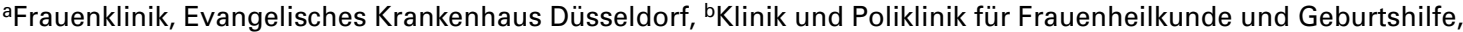 \\ Klinikum Großhadern, Ludwig-Maximilians-Universität München
}

\section{Schlüsselwörter}

Ovarialkarzinom · Rezidivchirurgie $\cdot$ Second-line-

Therapie $\cdot$ Third-line-Therapie

\section{Zusammenfassung}

Kommt es nach der Primärtherapie des Ovarialkarzinoms zu einem Rezidiv, so ist eine erneute Operation nur unter günstigen Voraussetzungen sinnvoll und erfolgversprechend. Dazu zählen ein rezidivfreies Intervall von mindestens 6-12 Monaten sowie ein möglichst tumorfreier Zustand nach der Primärtherapie. Auch nach der Rezidivoperation ist die makroskopische Tumorfreiheit der prognostisch wichtigste Faktor. Diese kann selbst bei Mehrfachlokalisation in fast $30 \%$ der Fälle erreicht werden. Die Behandlungsergebnisse sind in onkologischen Zentren mit optimaler interdisziplinärer Zusammenarbeit weitaus besser als in kleineren Einrichtungen. Eine Chemotherapie kommt sowohl für die Konsolidierung nach erfolgreicher Rezidivoperation als auch als rein palliativer Ansatz bei inoperablen Rezidiven in Betracht. Seit Paclitaxel in die Primärtherapie übernommen wurde, gibt es für die Second-line-Chemotherapie keinen definierten Standard. Zahlreiche Zytostatika weisen in dieser Situation eine begrenzte Aktivität auf, darunter Treosulfan, Epirubicin und die neueren Substanzen Topotecan, Gemcitabin, Vinorelbin und PEG(Polyethylenglykol)-liposomales Doxorubicin. Die Arbeitsgemeinschaft Gynäkologische Onkologie (AGO) hat eine Reihe randomisierter Studien für die Rekrutierung geöffnet, durch die für die Second-line-Situation ein neuer Standard definiert werden soll.

\author{
Key Words \\ Ovarian cancer - Surgery in recurrent cancer . \\ Second-line therapy - Third-line therapy
}

\section{Summary}

Secondary surgery after failure of primary treatment is a promising and reasonable option only for patients with a relapse-free interval of at least 6-12 months who should have ideally achieved a tumor-free status after primary therapy. As after primary surgery, size of residual tumor is the most significant predictor of survival after secondary surgery. Even in the case of multiple tumor sites, complete removal of the tumor can be achieved in nearly $30 \%$ of the patients. Treatment results are much better in specialized oncology centers with optimal interdisciplinary cooperation compared with smaller institutions Chemotherapy can be used both for consolidation after successful secondary surgery and for palliation in patients with inoperable recurrent disease. Since paclitaxel has been integrated into first-line chemotherapy, there is no defined standard for second-line chemotherapy. Several cytotoxic agents have shown moderate activity in this setting, including treosulfan, epirubicin, and newer agents such as topotecan, gemcitabine, vinorelbine, and PEG(polyethylene glycol)-liposomal doxorubicin. Thus, the German Arbeitsgemeinschaft Gynäkologische Onkologie (AGO) has initiated several randomized studies in patients with recurrent ovarian cancer in order to define new standards for second-line chemotherapy.

\begin{tabular}{ll}
\hline KARGER & @ 2000 S. Karger GmbH, Freiburg \\
Fax +4976145207 14 & Accessible online at: \\
$\begin{array}{l}\text { E-mail Information@Karger.de } \\
\text { www.karger.com }\end{array}$ & www.karger.com/journals/onk
\end{tabular}




\section{Differenzierter Therapieansatz}

Der Stellenwert einer Rezidivbehandlung beim Ovarialkarzinom wird nach wie vor kontrovers diskutiert. Die jeweils extremen Standpunkte reichen von einer totalen Ablehnung jeglicher Therapie beim Rezidiv bis zu der Maßgabe, in jedem Fall nochmals auf die operative Option zu setzen. Beide Extreme sind in ihrer Absolutheit falsch, da sie die verschiedenen Ausgangssituationen prognostisch unterschiedlicher Rezidivgruppen nicht berücksichtigen. So überleben Patientinnen mit Progression unter oder kurz nach Abschluss der primären postoperativen Chemotherapie im Median nur zirka 6 Monate. Bei dieser Patientengruppe stehen rein palliative Therapieformen im Vordergrund, eine nochmalige Operation sollte nur bei Ileuszuständen erwogen werden. Meist handelt es sich hier um Patientinnen, die schon im operativen Erstansatz nicht tumorfrei operiert werden konnten, so dass eine Rationale für den operativen Zweitansatz, noch dazu bei Progression unter Chemotherapie, nicht ersichtlich ist. Im Gegensatz dazu überleben Patientinnen mit Spätrezidiv im Median 24 Monate, so dass hier sowohl ein operativer als auch ein chemotherapeutischer Ansatz möglich ist.

\section{Operative Option beim Rezidiv}

Bei Auftreten eines Ovarialkarzinom-Rezidivs stehen grundsätzlich verschiedene Therapiemöglichkeiten zur Verfügung: Erneute Operation, Chemotherapie, Hormontherapie, Radiatio, Immuntherapie, aber auch keine Therapie.

Ob die Rezidivchirurgie sinnvoll und erfolgversprechend ist, hängt von zahlreichen Faktoren ab. Unter den folgenden Voraussetzungen ist die Chance, dass eine Patientin aus einem erneuten operativen Eingriff einen substanziellen Nutzen zieht, relativ hoch:

- Initial günstige Prognoseparameter wie z.B. kein Tumorrest nach Primärchirurgie, frühes Stadium oder komplette Remission nach Chemotherapie bei Tumorrest

- Langer Abstand zwischen Primärtherapie und Rezidiv

- Tumorfreiheit bei Rezidivoperation wahrscheinlich.

Vor allem muss konsequent zwischen Patientinnen mit Frührezidiv (erneutes Auftreten der Krankheit innerhalb von zirka 6 Monaten nach Abschluss der postoperativen Chemotherapie) und dem prognostisch günstigeren Spätrezidiv unterschieden werden. Dabei ergibt sich mit zunehmendem zeitlichen Abstand des Rezidivs von der Primärtherapie eine bessere Prognose. Während in der ersten Gruppe nur ein geringer Prozentsatz $(<5 \%)$ makroskopisch tumorfrei operiert werden kann, gelingt dies bei Patientinnen mit Spätrezidiv in über $40 \%$ der Fälle. Dazu ist zu bemerken, dass die makroskopische Tumorfreiheit ebenso wie in der Primärtherapie den entscheidenden Prognosefaktor hinsichtlich des weiteren Überlebens darstellt. So betrug die mediane Überlebenszeit in unserem Patientenkollektiv bei tumorfrei operierten Patien- tinnen 43 Monate, bei Tumorrest bis $2 \mathrm{~cm} 24$ Monate und bei Tumorrrest über $2 \mathrm{~cm}$ lediglich 12 Monate. Ähnliche Unterschiede in den Überlebensdaten wurden von mehreren Autoren [1-5] angegeben, wobei die mediane Überlebenszeit bei makroskopisch tumorfrei operierten Frauen je nach Patientenzahl und Ausgangssituation zwischen 29 und 43 Monaten lag (Tab. 1).

Die erreichbare Tumorfreiheit hängt natürlich von der Lokalisation des Rezidivtumors ab. Bei ausschließlich im kleinen Becken lokalisierten Tumoren beträgt diese Rate bei unseren Patienten 57\%, weitere 29\% können bis auf einen Tumorrest bis $2 \mathrm{~cm}$ operiert werden. Aber auch bei Mehrfachlokalisation im gesamten Abdomen gelingt es bei fast $30 \%$ der Patientinnen, eine makroskopische Tumorfreiheit zu erzielen. Auch Lymphknoten-Rezidive können in einem hohen Prozentsatz erfolgreich reseziert werden.

Häufig ist für die Durchführung eines maximalen Debulkings inklusive Darmresektionen oder Blasenteilresektion eine optimale interdisziplinäre Zusammenarbeit zwischen Anästhesisten/Intensivmedizinern, Chirurgen, Internisten und Urologen erforderlich. Vor Durchführung des Eingriffs sollten die therapeutischen Optionen zwischen den einzelnen Disziplinen ausgiebig diskutiert werden. Wie Statistiken zeigen, kann in spezialisierten onkologischen Zentren bei $40-80 \%$ der Rezidivpatientinnen ein optimales Debulking erzielt werden, in den übrigen Krankenhäusern dagegen nur in 20-30\% der Fälle.

Die hohe Rate an makroskopisch tumorfreien Operationen ist jedoch nicht ohne entsprechenden Einsatz zu erzielen. So betrug die mediane Operationszeit bei unseren Patienten 250 (35-485) min, im Mittel mussten 2 Blutkonserven gegeben werden, bei einer Patientin waren 17 Konserven erforderlich. Die Hospitalisationsdauer betrug im Median 16 Tage bei einer Spannbreite von 7 bis 59 Tagen. Die Morbiditätsrate betrug $37 \%$. Dies erscheint sehr hoch, jedoch sind darin auch konservativ behobene Subileus-Zustände und Fieber über mehr als 2 Tage enthalten. Somit sind die Höhe der Morbidität und auch die Mortalität mit 2\% akzeptabel, wenn man die Schwere der Krankheitsbilder berücksichtigt, die Patientinnen mit Rezidiv eines Ovarialkarzinoms aufweisen.

\section{Chemotherapie}

Sowohl nach einer erfolgreich durchgeführten Rezidivchirurgie als auch im primären Ansatz spielt die Auswahl der Chemotherapie für die Lebensqualität der Patientinnen eine entscheidende Rolle. Während allerdings beim kurativen Therapieansatz die mögliche Heilung oder zumindest eine Verlängerung der rezidivfreien Überlebenszeit im Vordergrund steht und für dieses Ziel auch eine höhere Toxizität akzeptiert werden kann, so ist beim palliativen Ansatz die Nebenwirkungsrate so gering wie möglich zu halten.

Seit der Hinzunahme von Paclitaxel in die Primärtherapie ist der Standard für die Second-line-Therapie beim Ovarialkarzi- 
Tab. 1. Überlebenszeit nach Rezidivoperation des fortgeschrittenen Ovarialkarzinom

\begin{tabular}{llll}
\hline Autoren & $\begin{array}{l}\text { Anzahl der } \\
\text { Patientinnen }\end{array}$ & $\begin{array}{l}\text { Mediane Gesamt- } \\
\text { Überlebenszeit, Monate }\end{array}$ & $\begin{array}{l}\text { Mediane Überlebenszeit } \\
\text { Tumorrest 0, Monate }\end{array}$ \\
\hline Morris et al., 1989 [1] & 33 & 9,4 & $19,5^{*}$ \\
Jänicke et al., 1992 [2] & 30 & 18 & 29 \\
Vaccarello et al., 1995 [3] & 38 & 19 & 41 \\
Eisenkop et al., 1995 [4] & 36 & - & 43 \\
Meier und du Bois, 1998 [5] & 109 & 24 & 43 \\
\hline
\end{tabular}

*Tumorrest $<1 \mathrm{~cm}$.

Tab. 2. Zytostatika mit Wirksamkeit in der Second-line-Therapie des fortgeschrittenen Ovarialkarzinoms

\begin{tabular}{ll}
\hline Treosulfan & Topotecan \\
Etoposid & Gemcitabin \\
Ifosfamid & Vinorelbin \\
Epirubicin & Liposomales Doxorubicin \\
Cyclophosphamid & \\
\hline
\end{tabular}

Tab. 3. Ergebnisse der Phase-III-Studie mit Topotecan vs. Paclitaxel in der Second-line-Therapie des fortgeschrittenen Ovarialkarzinoms nach Versagen einer Platintherapie [7]

\begin{tabular}{lllll}
\hline Endpunkt & Topotecan & Paclitaxel & Risikoquotient & p-Wert \\
\hline Remissionsrate & $20,6 \%$ & $13,1 \%$ & - & 0,196 \\
$\quad$ Komplette Remission & $4,5 \%$ & $2,6 \%$ & - & - \\
$\quad$ Partielle Remission & $16,1 \%$ & $10,5 \%$ & - & - \\
Tumorstillstand & $29,5 \%$ & $33,3 \%$ & - & - \\
Remissionsdauer & 25,9 Wochen & 21,6 Wochen & 0,778 & 0,476 \\
Progressionsfreies Intervall & 18,9 Wochen & 14,7 Wochen & 0,764 & 0,072 \\
Überleben & 63,0 Wochen & 53,0 Wochen & 0,974 & 0,872 \\
Remissionsrate nach Crossover & \multirow{2}{*}{$10,2 \%$} & - & 0,638 \\
& $13,1 \%$ & & - & \\
\hline
\end{tabular}

nom nicht definiert. Mit Treosulfan, Etoposid und Epirubicin stehen, zusammen mit einer Vielzahl hier nicht aufgeführter Substanzen, Medikamente zur Verfügung, die in ihrer Wirkung beim Ovarialkarzinom seit langem bekannt sind. An neueren Substanzen sind Topotecan, Gemcitabin und das liposomale Doxorubicin zu erwähnen (Tab. 2).

Mit Treosulfan wurde in der Second-line-Therapie in Dosierungen von 5 oder $7 \mathrm{~g} / \mathrm{m}^{2}$ i.v. über $2 \mathrm{~h}$ mit Zykluswiederholung alle 4 Wochen eine Remissionsrate von $19 \%$ erzielt [6]. Von 80 auswertbaren Patientinnen gelangten 2 in eine komplette und 13 in eine partielle Remission, ein Tumorstillstand wurde in 27 Fällen (34\%) erreicht. Die Responder erreichten eine mediane Überlebenszeit von 41 Monaten, die Patientinnen mit Tumorstabilisierung von 18 Monaten und die Nonresponder von 5 Monaten. Die Nebenwirkungen der Therapie waren zumeist moderat. Lebensbedrohliche Myelosuppression, therapieresistente Übelkeit/Erbrechen oder Alopezie traten nicht auf. Insgesamt wurde die Lebensqualität der Patientinnen durch die Therapie kaum beeinträchtigt.

Auf der ASCO-Tagung 1998 wurden die Ergebnisse einer randomisierten Phase-III-Studie vorgestellt, in der Paclitaxel und Topotecan in der Second-line-Therapie des platinvorbehandelten Ovarialkarzinoms miteinander verglichen wurden [7]. Topotecan wurde in einer Dosierung von $1,5 \mathrm{mg} / \mathrm{m}^{2} /$ Tag als 30-minütige Infusion an 5 aufeinanderfolgenden Tagen alle 3 Wochen verabreicht (112 Patientinnen), Paclitaxel in einer Dosierung von $175 \mathrm{mg} / \mathrm{m}^{2}$ als 3-stündige Infusion alle 3 Wochen (114 Patientinnen). Die Patientinnen konnten nach Versagen ihrer randomisierten Behandlung auf die jeweils andere Studienmedikation umgestellt werden, was in etwa der Hälfte der Fälle auch geschah (61 Patientinnen auf Topotecan, 49 auf Paclitaxel). Tabelle 3 zeigt die Ergebnisse dieser Studie. Demnach zeigten sich in der Wirksamkeit beider Zytostatika keine signifikanten Unterschiede, das progressionsfreie Intervall 
Abb. 1. Randomisierungsschema der OVAR2.3-Studie (Second-line-Therapie).

Abb. 2. Randomisierungsschema der OVAR2.5-Studie. AUC $=$ area under the curve.

Abb. 3. Randomisierungsschema der OVAR2.3-Studie (Third-line-Therapie).
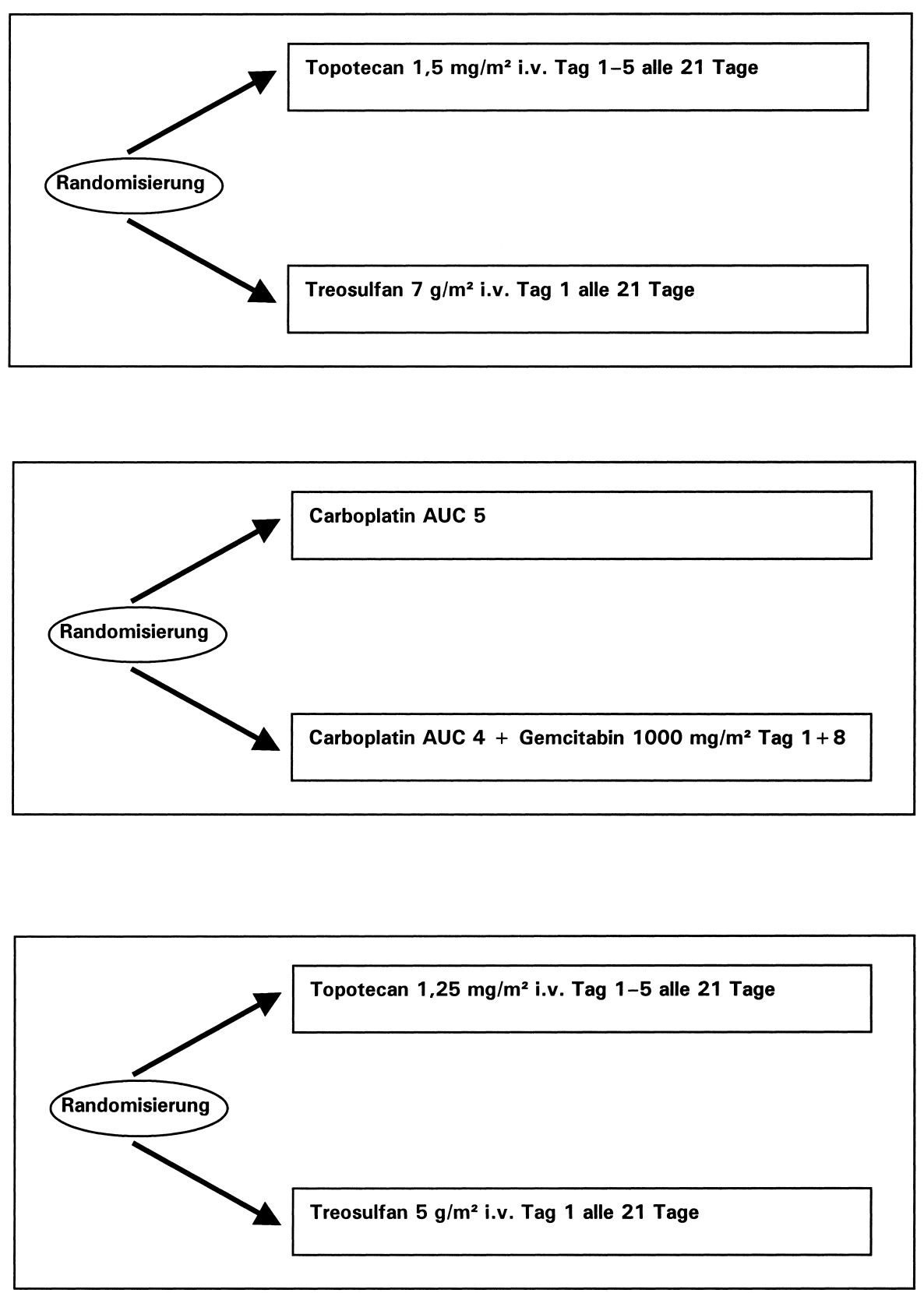

war nach Topotecan-Therapie sogar tendenziell länger. Topotecan war deutlich myelotoxischer als Paclitaxel (Grad-4-Neutropenie $36 \%$ vs. $9 \%$, Grad-4-Thrombozytopenie $10 \%$ vs. $<1 \%$, Grad-3/4-Anämie $16 \%$ vs. $2 \%$ ), während die nichthämatologische Toxizität in beiden Therapiearmen größtenteils gering ausgeprägt war.

Um ebenso wie in der Primärtherapie auch in der Secondline-Situation neue Standards zu definieren, hat die Studiengruppe Ovarialkarzinom der Arbeitsgemeinschaft Gynäkologische Onkologie (AGO) Studien initiiert, die in Abhängigkeit von der prognostischen Einschätzung verschiedene Einzelsubstanzen und Kombinationsregimes überprüfen. Einzelheiten zu diesen Studien können im Internet unter www.ago-ovar.de abgerufen werden.
Für die Second-line-Therapie von Patientinnen mit Progression unter der primären Therapie bzw. Frührezidiv bis 6 Monate nach Abschluss der initialen Platin/Paclitaxel-Behandlung (= platinrefraktäre Erkrankung) und von Patientinnen mit Rezidiv 6 und 12 Monate nach der initialen Behandlung (= intermediäres Rezidiv) wird Topotecan mit Treosulfan verglichen (OVAR 2.3) (Abb. 1).

Bei Patientinnen mit Spätrezidiv ( $>12$ Monate nach Platin/Paclitaxel), d.h. mit platinsensitiver Erkrankung, soll untersucht werden, ob eine Reinduktion mit Carboplatin alleine ebenso effektiv ist wie die Kombination aus Carboplatin und Gemcitabin (OVAR 2.5) (Abb. 2). In dieser Studie werden die Patientinnen danach stratifiziert, ob zuvor eine Rezidivoperation stattgefunden hat oder nicht. 
Tritt nach Platin-Reinduktion eine erneute Progression auf, können diese Patientinnen in die Studie der Third-line-Therapie mit Topotecan vs. Treosulfan, hier in reduzierter Dosis, eingebracht werden (Abb. 3).

\section{Fazit}

Zusammenfassend bleibt festzuhalten, dass abgesehen von individuellen Ausnahmen die Rezidivchirurgie nur bei Patien- tinnen mit Spätrezidiv zum Einsatz kommen sollte. Voraussetzung sollte die mögliche Erreichbarkeit einer makroskopischen Tumorfreiheit sein, die oftmals nur in interdisziplinärer Zusammenarbeit mit Chirurgie und Anästhesie erzielt werden kann. Ist diese nicht gegeben, sollte die Verlegung in ein entsprechend ausgerüstetes Zentrum erfolgen. Hinsichtlich der Chemotherapie sollten die zur Verfügung stehenden Substanzen im Rahmen prospektiv randomisierter Studien überprüft werden. Nur so wird eine weitere Verbesserung der Therapieergebnisse beim rezidivierten Ovarialkarzinom möglich sein.

\section{Literatur}

1 Morris M, Gershenson DM, Wharton JT: Secondary cytoreductive surgery in epithelial ovarian cancer: Nonresponders to first-line therapy. Gynecol Oncol 1989;33:1-5.

2 Jänicke F, Hölscher M, Kuhn W, von Hugo R, Pache L, Siewert JR, Graeff H: Radical surgical procedure improves survival time in patients with recurrent ovarian cancer. Cancer 1992;70:21292136.
3 Vaccarello L, Rubin SC, Vlamis V, Wong G, Jones WB, Lewis JL, Hoskins WJ: Cytoreductive surgery in ovarian carcinoma patients with a documented previously complete surgical response. Gynecol Oncol 1995;57:61-65.

4 Eisenkop SM, Friedman RL, Wang H: Secondary cytoreductive surgery for recurrent ovarian cancer. Cancer 1995;76:1606-1614.

5 Meier W, du Bois A: Palliative therapeutische Möglichkeiten beim Ovarialkarzinom. Onkologe 1998;4:1153-1158.
6 Gropp M, Meier W, Hepp H: Treosulfan as an effective second-line therapy in ovarian cancer. Gynecol Oncol 1998;71:94-98.

7 Gordon A, Carmichael J, Malfetano J, Gore M, Spaczynski M, Clarke-Pearson D, Ten Bokkel Huinink W, King K, Krebs JB, Fields SZ: Final analysis of a phase III, randomized study of topotecan (T) vs paclitaxel $(\mathrm{P})$ in advanced epithelial ovarian carcinoma (OC): International Topotecan Study Group. Proc Am Soc Clin Oncol 1998;17:1374(abstract). 